\title{
Network properties of mixtures of protonated and deuterated polyethylene
}

\author{
S. Kreitmeier and D. Göritz \\ Universität Regensburg, Regensburg, F.R.G.
}

\begin{abstract}
Mixtures of protonated and deuterated polyethylene were irradiated in the melt. The degree of crystallinity, the degree of crosslinking, as well as the enthalpy $\Delta H$ and the melting point $T_{M}$ were determined. No significant differences in the degree of crosslinking between protonated and deuterated chains were found. The mass specific entropy $\Delta S$ of the uncrosslinked samples remained constant and independent of the deuterium concentration. For the crosslinked samples, a netpoint entropy was postulated. A weaker Van der Waals interaction could explain the decrease in melting temperature by deuteration (for weakly crosslinked samples).
\end{abstract}

Key words: Protonated and deuterated polyethylene, mixtures, crosslinking, crystallinity, entropy, enthalpy.

\section{Introduction}

In recent years, the neutron scattering technique for investigating mixtures of protonated and deuterated polymers has advanced very much. However, some materials e. g. polyethylene $[1,2]$ show demixing phenomena, which complicate each analysis and render some entirely questionable. One way to avoid this demixing problem might be to crosslink the polymer in the melt, since homogeneous mixing is then ensured $[3,4]$. At low degrees of crosslinking, Schelten [3] was unable to achieve improvements. However almost nothing is known about the behaviour of such mixtures when they are crosslinked. For example, are there any preferences between proton and deuteron during the crosslinking process? Before starting any neutron scattering experiments [8], our aim was therefore to use highly crosslinked materials and to determine the calorimetric behaviour, the degree of crystallinity and the degree of crosslinking. Polyethylene was chosen for the investigation.

\section{Experimental}

\subsection{Sample material and preparation}

Linear protonated polyethylene $\left(M_{w}=600000 \mathrm{~g} / \mathrm{mol}, U=8\right)$ and matched deuterated polyethylene were mixed in o-xylene at $120^{\circ} \mathrm{C}$, precipitated in methanol and dried in vacuum. The resulting powder was then compression moulded at $150^{\circ} \mathrm{C}$ and in $\mathrm{N}_{2}$ atmosphere into sheets of about $1 \mathrm{~mm}$ thickness by applying a pressure of $10 \mathrm{kPa}$. The sheets were irradiated in the melt using a van de Graaff generator ( $2 \mathrm{MeV}$ electrons) (BASF, Ludwigshafen). Irradiation doses of $5 \mathrm{Mrad}, 20 \mathrm{Mrad}$ and $40 \mathrm{Mrad}$ were chosen. The samples were cooled in air. The degree of deuterium within the samples was 30\%, 50\% and 70\%. Samples of $0 \%$ and $100 \%$ served as references.

\subsection{Experimental methods}

\section{Degree of crystallinity}

To obtain the degree of crystallinity $\alpha$ a density gradient column containing propanol and glycol was used (cf. [9]).

\section{Degree of crosslinking}

The degree of crosslinking was estimated by swelling measurements in $\mathrm{o}$-xylene at $100^{\circ} \mathrm{C}$. The resulting elongation of the swollen isotropic sample was recorded by TMA (see Fig. 1).

\section{Enthalpy and melting point}

A Perkin Elmer DSC-2 was used to measure the enthalpy $\Delta H$ per gram and the melting point $T_{M}$. The heating rate was $5 \mathrm{~K} / \mathrm{min}$. To obtain the melting point, the peak value of the melting curve was chosen.

\section{Long spacing and crystallite size}

Kratky and Kiessig camera recordings were carried out to determine the distribution of the crystallite size and the long spacing. 


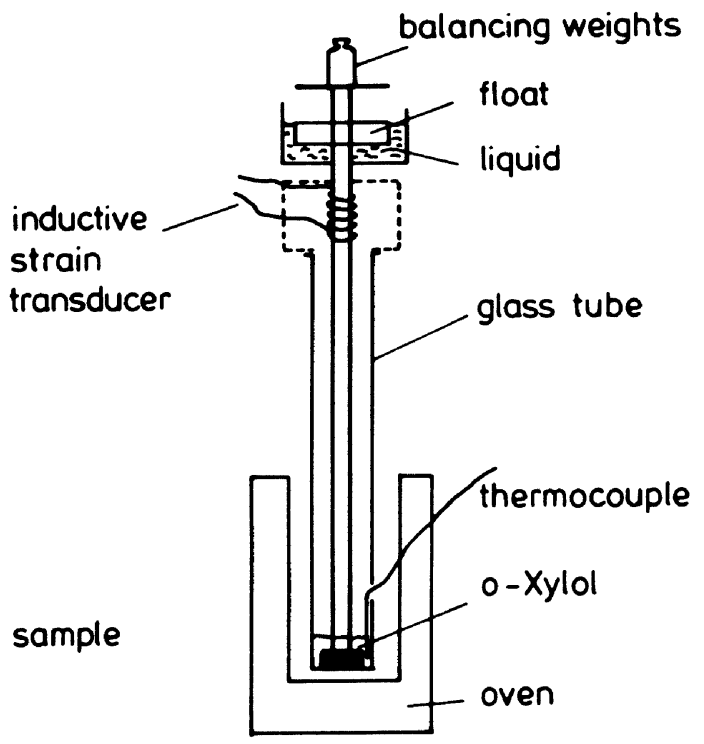

Fig. 1. TMA modified for swelling measurements

\subsection{Experimental results}

\section{Degree of crystallinity}

The results of the density measurements are shown in Fig. 2. In general, a decrease of crystallinity occurs with increasing radiation dose (compare with $[5,11]$ ). The increase of deuteration leads to a slightly higher degree of crystallinity compared to that of the pure protonated sample.

\section{Degree of crosslinking}

The Flory-Rehner equation was used to determine the crosslink density per unit volume from the volume changes measured by TMA. The interaction parameter was chosen as 0.43 [5]. The results converted to crosslink density per chain are shown in Fig. 3. It can be said that the degree of crosslinking of the samples, despite different deuteration, was not altered significantly. IR studies excluded the possible influence of $\mathrm{H}-\mathrm{D}$ exchange.

\section{Enthalpy and melting point}

Figure $4 \mathrm{a}$ shows the DSC curves of the $50 \%$ deuterated sample. The melting points decrease with increasing crosslinking, whereas the melting range broadens significantly. In Fig. 5, the resulting melting temperatures are plotted versus the concentration of deuterium; in Fig. 6 the values of the enthalpy versus

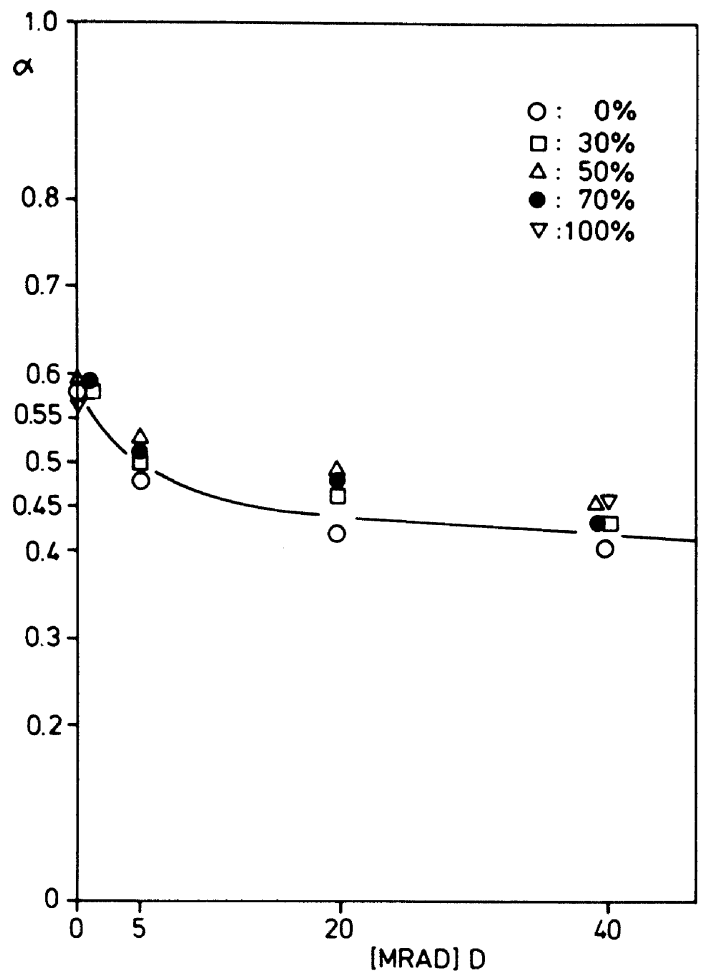

Fig. 2. Degree of crystallinity $\alpha$ versus irradiation dose D. Parameter: concentration of deuterium $c$

concentration are shown. Figure $4 \mathrm{~b}$ shows the DSC curves of the 40 Mrad irradiated samples which will be discussed separately.

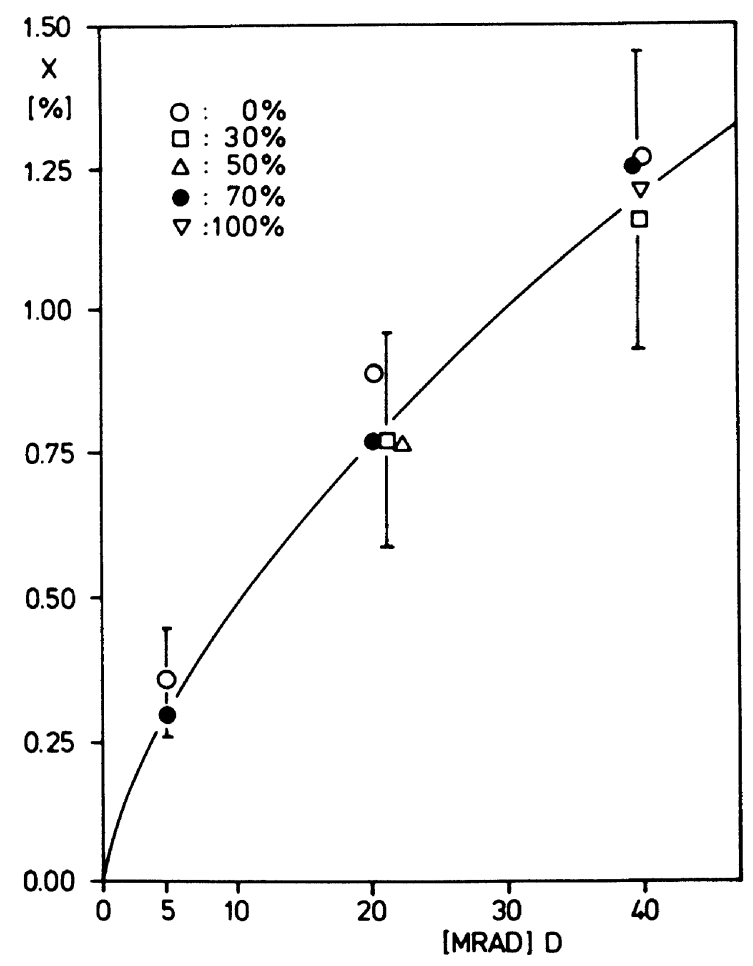

Fig. 3. Crosslinks per chain $X$ versus irradiation dose $D$. Parameter: concentration of deuterium $c$ 


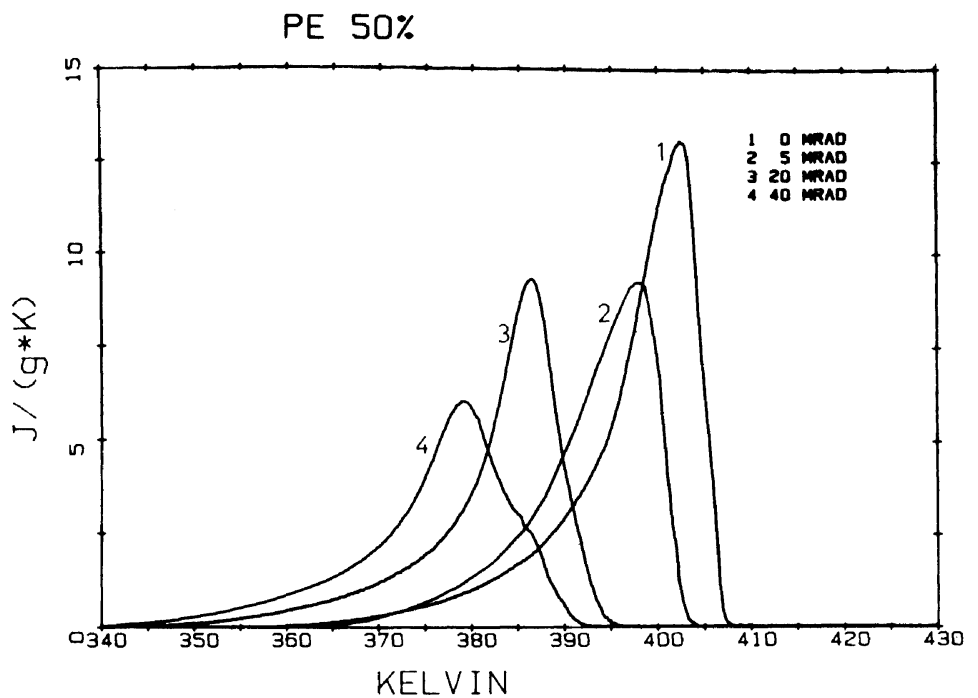

Fig. 4 a. DSC plots of the $50 \%$ deuterated samples. Parameter: irradiation dose $D$

\section{Long spacing and crystallite size}

Table 1 lists the long spacings determined by analysing the Kiessig camera photographs. The listed values are the averages for the different mixtures with a fixed degree of crosslinking. Within one mixture series, the long spacing slightly decreases with deuteration. The counts versus scattering vector for smeared Kratky

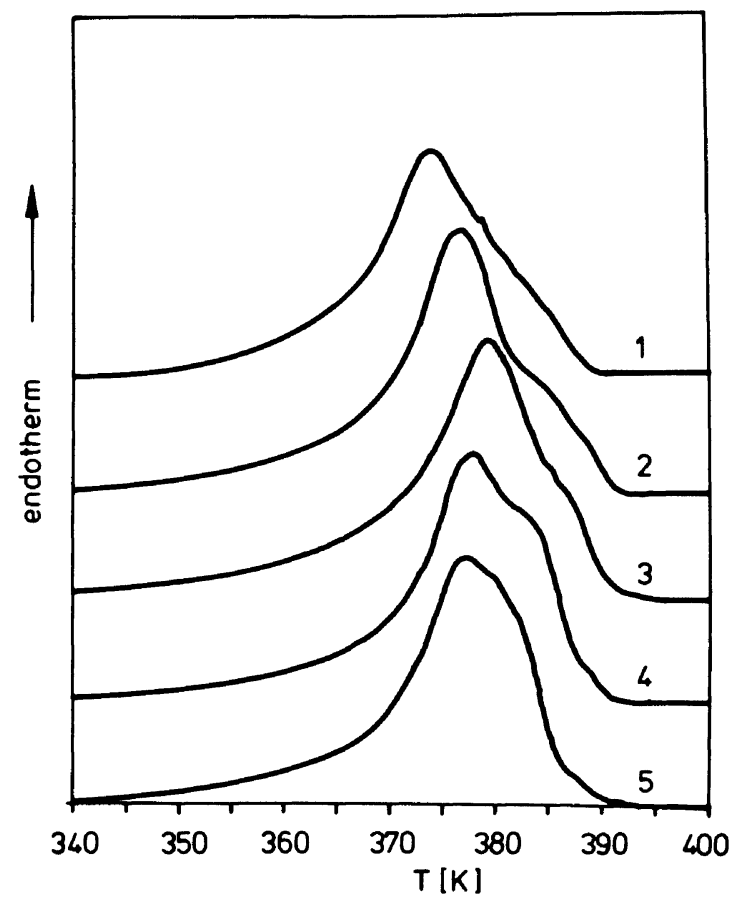

Fig. 4 b. DSC plots of the 40 Mrad irradiated samples. Parameter: concentration of deuterium c. (1) $0 \%$; (2) $30 \%$; (3) $50 \%$; (4) $70 \%$; (5) $100 \%$

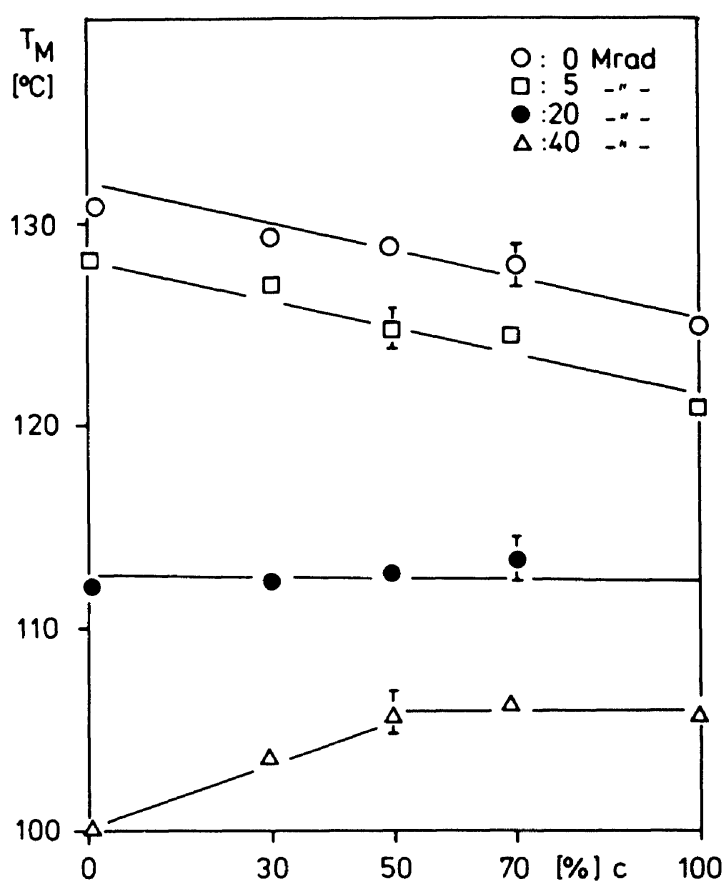

Fig. 5. Melting temperature $T_{M}$ versus concentration of deuterium c. Parameter: irradiation dose $D$

curves of the $50 \%$ deuterium mixture are shown in Fig. 7. With increasing crosslinking, the scattering curves get flatter and broader. All other mixtures behave similarly.

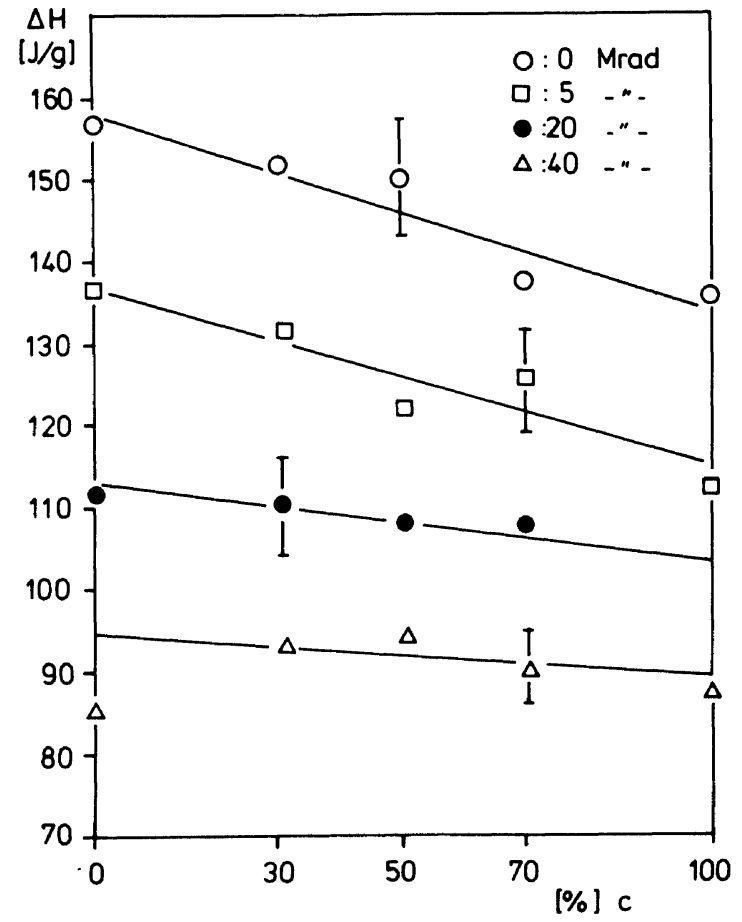

Fig. 6. Specific enthalpy $\Delta H$ versus concentration of deuterium $c$. Parameter: irradiation dose $D$ 
Table 1. Long spacings determined from analysis of Kiessig photographs

\begin{tabular}{ll}
\hline irradiation dose $D$ & long spacing $L$ \\
\hline $0 \mathrm{Mrad}$ & $400 \AA$ \\
$5 \mathrm{Mrad}$ & $340 \AA$ \\
$20 \mathrm{Mrad}$ & $250 \AA$ \\
$40 \mathrm{Mrad}$ & $180 \AA$ \\
\hline
\end{tabular}

\section{Discussion}

\section{Crystallite size}

The degree of crystallinity decreases with crosslinking (cf. Fig. 3). On the other hand, the long spacing also decreases (see Table 1) and the smeared Kratky recordings show a flatter and broader curve when raising the degree of crosslinking. Together with the results of Jäger et al. [5] these let us suppose that there is a large decrease in crystallite size combined with an increase in size distribution. Smaller crystallites, i. e. lamellae which decrease, especially laterally (cf. [5]), enable a larger proportion of amorphous contributions by decreasing crystallintiy and long spacing. This statement is strengthened by the DSC measurements. The decreasing melting point and the increasing melting range also point to smaller crystallites and a greater variety in crystallite size.

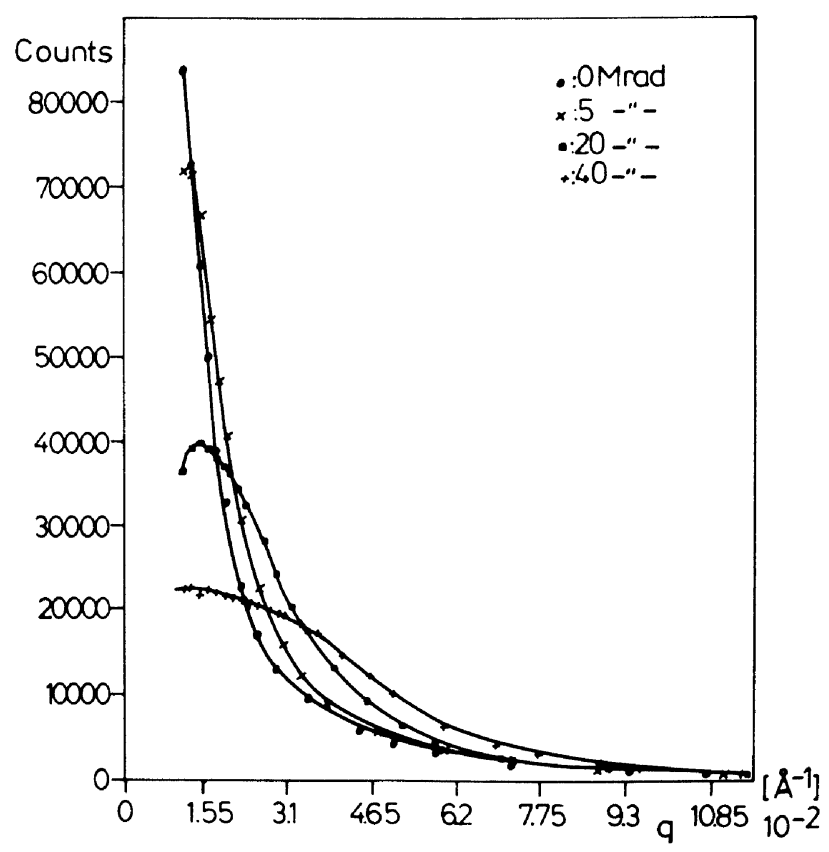

Fig. 7. Counts versus scattering vector $q$ of the $50 \%$ deuterated sample

\section{Crosslinking}

The crystallinity and the swelling measurements allow us to assume that crosslinking of both protonated and deuterated material leads to a similar degree of crosslinking.

\section{Entropy: uncrosslinked samples}

The values of the melting entropy were calculated according to $\Delta S=\Delta H / T_{M}$. To obtain the specific entropy, the mass ratio of the monomers $m_{H} / m_{D}=7 / 8$ must be taken into account. Using

$$
\Delta S^{\mathrm{corr}}=(1-c) \cdot \Delta S^{\mathrm{meas}}+c \cdot 8 / 7 \cdot \Delta S^{\mathrm{meas}}
$$

to calculate the melting entropy, where $c$ is the concentration of deuterium, the results in Fig. 8 are obtained. The specific entropy remains constant despite different deuterium concentrations. This is not surprising since the geometry of the PE molecule is virtually unaltered by deuteration. Therefore, the difference of about $6 \mathrm{~K}$ in melting temperature, which has also been found by other authors $[6,7]$, has to be explained by changes in melting enthalpy. One possibility might be a weaker Van der Waals interaction of deuterons in crystals. The attracting part of the Van der Waals interaction results from dipole-dipole interaction of the sharing atoms. To a first order approximation one may restrict the problem to $\mathrm{H}-\mathrm{H}, \mathrm{H}-\mathrm{D}$ and $\mathrm{D}-\mathrm{D}$ interactions. The resulting ground state is proportional to $\omega_{o}=\sqrt{\frac{C}{\mu}}[10]$, with force constant $C$ and reduced mass $\mu$. With the reduced masses

$$
\mu_{H}=\frac{m}{1+\frac{m}{M}} \quad \mu_{D}=\frac{m}{1+\frac{m}{2 M}}
$$

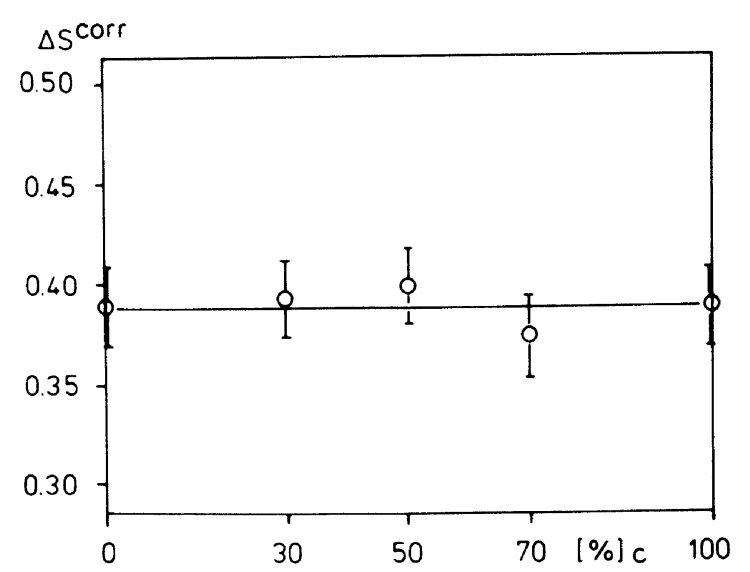

Fig. 8. Specific entropy $\Delta S^{\mathrm{cor}}$ (units in $\mathrm{J} / \mathrm{K}$ and equivalent mass) versus concentration of deuterium $c$, uncrosslinked samples 
where $m=$ electron mass, $M=$ proton mass, a slightly weaker dipole-dipole attraction arises for the D-D interaction. The repulsion term follows from the orbital overlapping. Filled orbitals force the sharing atoms into disadvantageous energy states. Taking the energy states

$$
\begin{aligned}
& E_{n}^{D}=-\frac{\mu_{D} e^{4}}{32 \pi^{2} \varepsilon_{o} \hbar^{2}} \cdot \frac{1}{n^{2}} \\
& E_{n}^{H}=-\frac{\mu_{H} e^{4}}{32 \pi^{2} \varepsilon_{o} \hbar^{2}} \cdot \frac{1}{n^{2}}
\end{aligned}
$$

where $n=$ main quantum number, $e=$ electron charge, $\varepsilon_{o}=$ dielectric constant, $\hbar=$ Planck's constant devided by $2 \pi$, into account, there results a greater energy gap to the next orbital for the $\mathrm{D}-\mathrm{D}$ interaction. Therefore, the repulsion for $\mathrm{D}-\mathrm{D}$ will be strengthened. On the assumption that the equilibrium distance of $\mathrm{H}$ $\mathrm{H}$ and $\mathrm{D}-\mathrm{D}$ atom pairs does not change (inflexible $\mathrm{C}-\mathrm{C}$ bonds may stabilize this) a weaker Van der Waals interaction results for the deuteron from the above. The $\mathrm{H}$ $\mathrm{D}$ interaction is, of course, situated in between.

\section{Entropy: crosslinked samples}

In the case of uncrosslinked samples, the measured values, corrected for monomer mass, show a constant melting entropy. This constance of the melting entropy, postulated for samples of fixed degree of crosslinking, together with the assumption that a minor deviation in the degree of crosslinking can be neglected, demands a constant (not mass dependent) term which must be substracted from the measured values. Only then is the constance of entropy according to Eq. (1) able to be achieved.

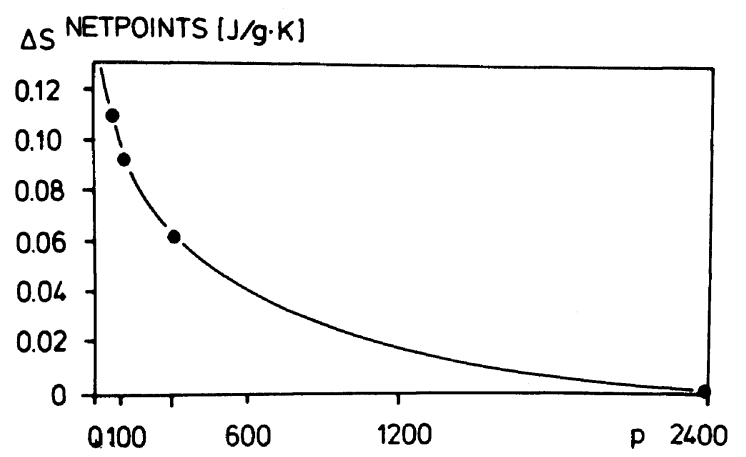

Fig. 9. Netpoint entropy $\Delta S^{\text {NETPOINTS }}$ versus degree of polymerisation of subchains $p$
Table 2. Netpoint entropy $\Delta S^{\text {NETPOINTS }}$ values (see text)

\begin{tabular}{lllll}
\hline Dose & $\Delta S_{0 \%}^{\text {INT. OF AXIS }}$ & $\Delta S_{100 \%}^{\text {INT. OF AXIS }}$ & $\Delta S^{\text {NETPOINTS }}$ & $\frac{\Delta S_{\text {prot }}}{\Delta S_{\text {deut }}}$ \\
\hline $0 \mathrm{Mrad}$ & 0.388 & 0.339 & 0.00 & $\cong \frac{8}{7}$ \\
$5 \mathrm{Mrad}$ & 0.340 & 0.305 & 0.060 & $=\frac{8}{7}$ \\
$20 \mathrm{Mrad}$ & 0.292 & 0.267 & 0.092 & $=\frac{8}{7}$ \\
$40 \mathrm{Mrad}$ & 0.255 & 0.237 & 0.111 & $=\frac{8}{7}$ \\
\hline
\end{tabular}

The values of $\Delta S^{\text {NETPOINTS }}$ were achieved by using formula

$$
\frac{\Delta S_{0 \%, \text { D Mrad }}^{\text {INTERCEP OF AXIS }}-\Delta S^{\text {NETPOINTS }}}{\Delta S_{100 \%, \text { D Mrad }}^{\text {INTER AXIS }}-\Delta S^{\text {NETPOINTS }}} \cdot
$$

The resulting values are listed in Table 2 and shown in Fig. 9. The term which is independent of mass is interpreted as a netpoint entropy. The incorporation of crosslinks into the crystallite gives this contribution

$$
\Delta S=\Delta S^{\mathrm{PROT}}+\Delta S^{\mathrm{DEUT}}+\Delta S^{\mathrm{NETPOINTS}} .
$$

The existence of a netpoint entropy corresponds to the conclusions obtained by Jäger et al. [5] in their experiments with pure protonated polyethylene.

\section{Crosslinks and crystallites}

From the results of Jäger et al. [5], up to $1 \%$ of crosslinks can be incorporated into the crystal. We may compare this with Fig. 10, which shows the degree $X_{c}$ of the incorporated crosslinks versus the total degree $X$ of crosslinks. With this in mind, the deviations in the melting temperature of the 40 Mrad purely protonate sample and of the $40 \mathrm{Mrad} 30 \%$ deuterated sample can be understood. Both are situated above $1 \%$ crosslink fraction (cf. Fig. 3). In this case, crosslinks will be expelled and micellar crystallites will be formed. The absence of this fact for the higher deuterated samples of the $40 \mathrm{Mrad}$ series could also be explained by the weaker Van der Waals interaction. These crystallites are possibly able to incorporate more crosslinks. The bimodal structure of the melting curves in Fig. 4 b may thus be understood. 


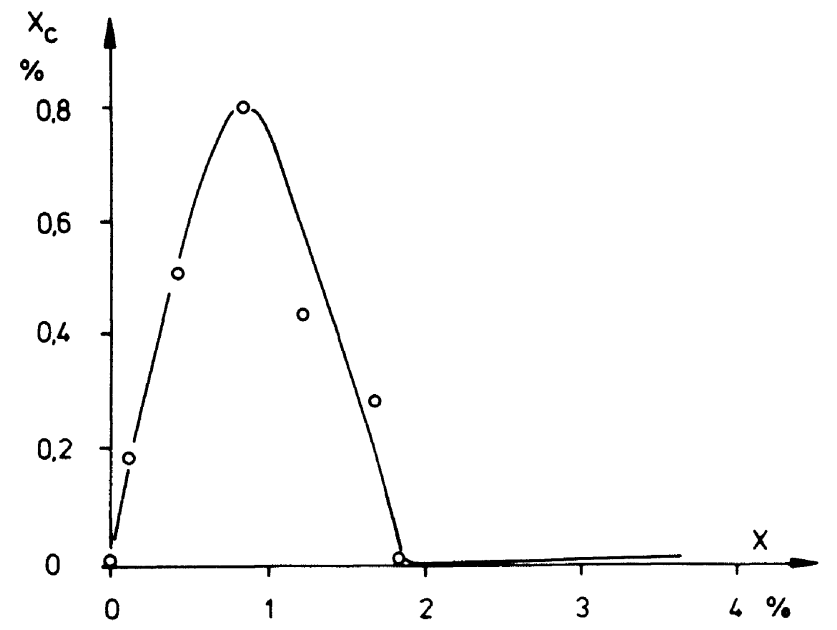

Fig. 10. Degree of incorporated crosslinks $X_{c}$ versus total degree of crosslinks $X$ from Ref. [5]

\section{Final remarks}

The calorimetric investigations on crosslinked samples have shown that the deuteration technique is useful not only for neutron scattering, but also for other experimental methods. In our case, the small differences between proton and deuteron allowed us to separate the netpoint entropy from the overall entropy changes due to crosslinking.

\section{References}

1. Schelten J, Ballard DGH, Wignall GD, Longman G, Schmatz W (1976) Polymer 17:751

2. Buckingham AD, Hentschel HGE (1980) J Polym Sci 18:853

3. Schelten J, Zinken A, Ballard DGH (1981) Coll Polym Sci 259:260

4. Sperling LH, Fernandez AM, Wignall GD (1984) ACS Symp Ser 243:71

5. Jäger E, Müller J, Jungnickel B-J (1985) Prog Coll Polym Sci 71:145

6. Schelten J, Wignall GD, Ballard DGH, Longman GW (1977) Polymer 18:111

7. Stehling FC, Ergos E, Mandelkern L (1971) Macromol 4:672

8. Kreitmeier S, Göritz D, in preparation

9. Angerer G (1971) Kolloid Z 245:1, 369-381

10. Kittel C (1983) Einführung in die Festkörperphysik, Oldenburg, München, Wien

11. Karttunen $M$, Törmälä $P$ (1984) Thermochim Acta 72:41-44

Received August 3, 1987; accepted October 26, 1987

Authors' address:

D. Göritz

Institut für Angewandte Physik

Universität Regensburg

Universitätsstr. 31

D-8400 Regensburg, F.R.G. 